\section{Plan Curricular y la Formación por Competencias en Estudiantes de Administración - UNMSM}

\author{
Curricular Plan and Training by Competences in \\ Administration Students - UNMSM
}

\begin{abstract}
RESUMEN
Nuestra investigación busca establecer una relación entre dos variables, el Plan Curricular y la Formación por Competencias de estudiante en la Escuela Profesional de Administración de la Facultad de Ciencias Administrativas (FCA) de la Universidad Nacional Mayor de San Marcos (UNMSM), dicha investigación fue realizada durante los años 2017 y 2018. El plan curricular, es el norte de todo proceso educativo en cualquier universidad; por otro lado, el mercado exige que los estudiantes por egresar tengan las suficientes competencias para enfrentarse no solo al mercado laboral, sino a la vida misma. Consideramos a la universidad como una escuela donde los estudiantes adquieren competencias las mismas que deberán ser progresivas conforme avanzan los módulos por ciclo. La presente investigación está tipificada como básica, primaria, descriptiva, correlacional, cuantitativa, bivariable y transaccional. La muestra que se obtuvo es de 80 estudiantes, utilizando un software estadístico se llegó a la conclusión de que existe una relación moderada entre el plan curricular y la formación por competencias.
\end{abstract}

Palabras claves: Currículo; plan curricular; competencias y enseñanza.

\begin{abstract}
Our research seeks to establish a relationship between two variables, the Curriculum Plan and the Student Competency Training at the Professional School of Administration of the National University of San Marcos, this research was carried out during the years 2017-2018. The curricular plan is the north of all educational process in any university; On the other hand, the market requires that the students to graduate have sufficient competencies to face not only the labor market, but life itself. We consider the university as a school where students acquire the same competences that must be progressive as the modules progress through the cycle. The present investigation is typified as basic, primary, descriptive, correlational, quantitative, bivariate and transactional. The sample that was obtained is 80 students, using statistical software it was concluded that there is a moderate relationship between the curriculum plan and training by competencies.
\end{abstract}

Keywords: Curriculum; curricular plan; competences and teaching.

\section{Félix Armando Rivera León ${ }^{1}$}

friveral@unmsm.edu.pe

\section{Jorge Luis Alarcón Cerna ${ }^{1}$}

jorge.alarcon@unmsm.edu.pe

${ }^{1}$ Universidad Nacional Mayor de San Marcos, Facultad de Ciencias Administrativas. Lima, Perú

Presentado: 04/11/2019 - Aceptado: 27/05/2020 - Publicado: 20/10/2020

(C) Los autores. Este artículo es publicado por Gestión en el Tercer Milenio de la Facultad de Ciencias Administrativas de la Universidad Nacional Mayor de San Marcos. Este es un artículo de acceso abierto, distribuido bajo los términos de la licencia Creative Commons Atribucion - No Comercia_Compartir Igual 4.0 Internacional. (http://creativecommons.org/licenses/by-nc-sa/4.0/) que permite el uso no comercial, distribución y reproducción en cualquier medio, siempre que la obra original sea debidamente citada. 


\section{INTRODUCCIÓN}

La investigación responde a la necesidad de cómo plantear un currículo para un programa universitario. Para este propósito se ha evaluado la bibliografía correspondiente. Si bien cada institución educativa tiene diferentes necesidades y características particulares, trataremos el currículo como un instrumento para dirigir el planeamiento de la enseñanza-aprendizaje en la universidad.

Asimismo, esta investigación plantea una solución al problema de cómo se relaciona el plan curricular con la formación por competencias en la Escuela Profesional de Administración de la UNMSM. Ante esta situación, hemos propuesto como hipótesis de manera directa y significativa la relación entre el plan curricular y la formación por competencias de los estudiantes en la Escuela Profesional de Administración de la Universidad Nacional Mayor de San Marcos, en los años 2017 y 2018.

Los antecedentes nacionales sobre esta investigación concluyen según Mejía (2015) que "existe una correlación significativa entre las variables plan curricular (el currículo en acción) y el desempeño académico por competencias" (p. 173). El autor resalta que se puede realizar una retroalimentación que puede modificar el currículo por motivo de las exigencias del mercado laboral tan dinámico y tan cambiante como el nuestro. Alejos y Sánchez (2015) plantean que "existe una correlación mutua y viceversa entre las variables plan curricular y perfil profesional. Los autores proponen un diagnóstico situacional del área de acción del profesional egresado" (p. 126). Lovatón (2011) concluye que "la herramienta diagnóstico situacional sirve para saber en qué situación se encuentra la formación de profesionales, a partir del diagnóstico, planteamos el currículo" (p. 97). Todos los autores concluyen que el currículo es un documento de guía que las entidades educativas usan para formar a los estudiantes según el perfil profesional de egreso que la entidad propone.

Isabell (2014) menciona que "los antecedentes internacionales sobre la investigación concluyen, el conocimiento de la calidad y duración del programa habría sido fundamental para interpretar los hallazgos longitudinales" (p.77). Existe, pues, a propuesta de la autora, la duración más larga de los programas, tiene una relación más fuerte con las competencias generadas por el participante. Por último, Bycroft (2011) llega a las siguientes conclusiones: "estas competencias se pueden dividir en las siguientes cuatro áreas: competencias personales, competencias de conocimiento y habilidad, competencias situacionales y organizacionales y competencias de metodología" (p. 231). Hay un grupo central dedicado de líderes en el campo de la investigación que conocen y pueden articular las competencias que los investigadores necesitan para llevar a cabo actividades de seguridad, con conocimiento y efectividad al tiempo que protegen el bienestar del participante de la investigación.

El modelo de Ayes (2003) indica que "las bases teóricas del plan curricular contemplan las dimensiones del currículo: académica (fundamentos y enfoques), laboral (ejecución y principios y metodológica (modelo y diseño)" (p. 3).

Las bases teóricas de la formación por competencias según Larraín y González (2007), "son clasificadas de distinta manera por diversos autores. Las que concitan el mayor grado de consenso son competencias básicas o instrumentales, competencias genéricas o transversales o intermedias o generativas o generales y las competencias específicas o técnicas o especializadas" (p. 10).

\section{MÉTODOS}

En la presente investigación los instrumentos utilizados que se implementaron fueron los cuestionarios, los cuales se validaron y tienen una alta confiabilidad. Estas herramientas se usaron para recabar información. La técnica utilizada es la encuesta, la cual en casi la totalidad de las preguntas de los instrumentos poseen una escala de valoración (Likert). La población del décimo ciclo es de 222 estudiantes de Administración, por ser el segmento supuestamente preparado a ser egresado de la Facultad; de los que se tomó una muestra de 80 estudiantes.

En el análisis estadístico se utilizó el software SPSS 23, el cual tiene por objetivo el análisis de datos que da como resultado muestras figuras y tablas. Para la contrastación de la 
hipótesis se utilizó el coeficiente de correlación de Spearman, para variables no paramétricas.

Según la tabla 1, la validez y confiabilidad de los instrumentos se realizó de la siguiente manera, validez de los instrumentos: Mediante juicio de expertos, y confiabilidad: Mediante la prueba de Alfa de Cronbach.

Tabla 1.

Análisis de confiabilidad del plan curricular

\begin{tabular}{lccc} 
& N & Ítems & Alfa de Cronbach \\
Plan curricular & 45 & 58 & 0,788 \\
\hline
\end{tabular}

Nota. Elaboración propia.

Se observa dentro del análisis de consistencia interna «Alfa de Cronbach» del instrumento plan Curricular, existe un nivel de confiabilidad aceptable $(\alpha=0.788)$ en cada uno de los instrumentos.

En la tabla 2, se encontró dentro del análisis de consistencia interna "Alfa de Cronbach» del instrumento formación por competencias, existe un buen nivel de confiabilidad aceptable $(\alpha=0.876)$. Por ello, se realizó una medición objetiva de la característica de interés en el presente estudio.

Tabla 2.

Análisis de confiabilidad de la formación por competencias

\begin{tabular}{lccc} 
& N & Ítems & Alfa de Cronbach \\
\hline $\begin{array}{l}\text { Desarrollo de } \\
\text { competencias }\end{array}$ & 45 & 26 & 0,876 \\
\hline
\end{tabular}

Nota. Elaboración propia.

\section{RESULTADOS}

En la tabla 3 , se observa que el $68.8 \%$ de los estudiantes presentan un nivel medio respecto a la percepción al plan curricular y el 31.3\% presenta un nivel bajo respecto al plan curricular.

Tabla 3.

Valoración del plan curricular

\begin{tabular}{lcc} 
& Frecuencia & Porcentaje \\
Bajo & 25 & 31,3 \\
Medio & 55 & 68,8 \\
Alto & 0 & 0,0 \\
Total & 80 & 100,0 \\
\hline
\end{tabular}

Nota. Elaboración propia

En la tabla 4, se observa que $91.3 \%$ de los estudiantes presentan un nivel medio respecto a la percepción del aspecto académico del plan curricular, el 5\% presenta un nivel bajo y el $3.8 \%$ presenta un nivel alto respecto del aspecto académico del plan curricular.

\section{Tabla 4.}

Valoración de dimensión de aspectos académicos

\begin{tabular}{lcc} 
& Frecuencia & Porcentaje \\
Bajo & 4 & 5,0 \\
Medio & 73 & 91,3 \\
Alto & 3 & 3,8 \\
Total & 80 & 100,0 \\
\hline
\end{tabular}

Nota. Elaboración propia

En la tabla 5, se observa que el $56.3 \%$ presenta un nivel bajo respecto a la formación laboral del plan curricular, el $42.5 \%$ de los estudiantes presentan un nivel medio respecto a la percepción de la formación laboral del plan curricular y el $1.3 \%$ presenta un nivel alto respecto a la formación laboral del plan curricular.

\section{Tabla 5.}

Valoración de dimensión de formación laboral

\begin{tabular}{lcc} 
& Frecuencia & Porcentaje \\
Bajo & 45 & 56,3 \\
Medio & 34 & 42,5 \\
Alto & 1 & 1,3 \\
Total & 80 & 100,0 \\
\hline
\end{tabular}

Nota. Elaboración propia

En la tabla 6, se observa que el $87.5 \%$ de los estudiantes presentan un nivel bajo respecto a los aspectos metodológicos al plan curricular y el $12.5 \%$ presenta un nivel medio.

\section{Tabla 6.}

Valoración de dimensión de aspectos metodológicos

\begin{tabular}{lcc} 
& Frecuencia & Porcentaje \\
Bajo & 70 & 87,5 \\
Medio & 10 & 12,5 \\
Alto & 0 & 0,0 \\
Total & 80 & 100,0 \\
\hline
\end{tabular}

Nota. Elaboración propia

En la tabla 7, se observa que el $71.3 \%$ de los estudiantes perciben un nivel bajo respecto a la formación por competencias y el $28.8 \%$ un nivel medio respecto a la formación por competencias. 
Tabla 7.

Formación por competencias

\begin{tabular}{lcc} 
& Frecuencia & Porcentaje \\
Bajo & 57 & 71,3 \\
Medio & 23 & 28,8 \\
Alto & 0 & 0,0 \\
Total & 80 & 100,0 \\
\hline
\end{tabular}

Nota. Elaboración propia

En la tabla 8, se observa que el 55\% de los estudiantes perciben un bajo nivel respecto a la formación por competencias básicas y el $45 \%$ un nivel medio respecto a la formación por competencias básicas.

Tabla 8.

Valoración de dimensión y formación por competencias básicas

\begin{tabular}{lcc} 
& Frecuencia & Porcentaje \\
Bajo & 44 & 55,0 \\
Medio & 36 & 45,0 \\
Alto & 0 & 0,0 \\
Total & 80 & 100,0 \\
\hline
\end{tabular}

Nota. Elaboración propia.

En la tabla 9, observamos que el $57.5 \%$ de los estudiantes perciben nivel bajo respecto a la formación por competencias genéricas y el $42.5 \%$ un nivel medio respecto a la formación por competencias genéricas.

Tabla 9.

Valoración de dimensión de formación por competencias genéricas

\begin{tabular}{lcc} 
& Frecuencia & Porcentaje \\
Bajo & 46 & 57,5 \\
Medio & 34 & 42,5 \\
Alto & 0 & 0,0 \\
Total & 80 & 100,0 \\
\hline
\end{tabular}

Nota. Elaboración propia.

En la tabla 10, se observa que el $87.5 \%$ de los estudiantes percibe nivel bajo respecto a la formación por competencias específicas y el $12.5 \%$ un nivel medio respecto a la formación por competencias específicas.

Tabla 10.

Dimensión de formación por competencias específicas

\begin{tabular}{lcc} 
& Frecuencia & Porcentaje \\
Bajo & 70 & 87,5 \\
Medio & 10 & 12,5 \\
Alto & 0 & 0,0 \\
Total & 80 & 100,0 \\
\hline
\end{tabular}

Nota. Elaboración propia.

\section{Hipótesis 1.}

H1a: Existe relación significativa entre los "aspectos académicos del plan curricular con la formación por competencias básicas" de "estudiantes del décimo ciclo de la Escuela Profesional de Administración", UNMSM, 2017-2018.

H1a0: No existe relación significativa entre los "aspectos académicos del plan curricular con la formación por competencias básicas" en los "estudiantes del décimo ciclo de la Escuela Profesional de Administración", UNMSM, 2017-2018.

\section{Contrastación de Hipótesis 1 (ver tabla 11).}

Tabla 11.

Correlación: "aspectos académicos y las competencias básicas"

\begin{tabular}{llc} 
& Coeficiente de correlación & $\begin{array}{c}\text { Competencias } \\
\text { básicas }\end{array}$ \\
Aspectos & Spearman & 0,390 \\
\cline { 2 - 3 } académicos & Sig. (bilateral) & 0,000 \\
\cline { 2 - 3 } & $\mathrm{N}$ & 80
\end{tabular}

Nota. Elaboración propia.

\section{Hipótesis 2.}

H2a: Existe una relación significativa entre la "formación laboral del plan curricular" con la "formación por competencias básicas" de "estudiantes del décimo ciclo de la Escuela Profesional de Administración", UNMSM, 2017-2018.

H2a0: No existe relación significativa entre la "formación laboral del plan curricular" con la "formación por competencias básicas" en los "estudiantes del décimo ciclo de la Escuela Profesional de Administración", UNMSM, 2017-2018.

\section{Contrastación de Hipótesis 2 (ver tabla 12).}

\section{Tabla 12.}

Correlación: "formación laboral y las competencias básicas"

\begin{tabular}{llc} 
& & $\begin{array}{c}\text { Competencias } \\
\text { básicas }\end{array}$ \\
Formación & $\begin{array}{l}\text { Coeficiente de correlación } \\
\text { Labearman }\end{array}$ & 0,158 \\
\cline { 2 - 3 } & Sig. (bilateral) & 0,162 \\
\cline { 2 - 3 } & $\mathrm{N}$ & 80
\end{tabular}

Nota. Elaboración propia.

\section{Hipótesis 3.}

H3a: Existe relación significativa entre los "aspectos metodológicos del plan curricular" con la "formación por competencias básicas" 
de "estudiantes del décimo ciclo de la Escuela Profesional de Administración", UNMSM, 20172018.

H3a0: No existe relación significativa entre los "aspectos metodológicos del plan curricular" " con la "formación por competencias básicas" de "estudiantes del décimo ciclo de la Escuela Profesional de Administración", UNMSM, 20172018.

Contrastación de Hipótesis 3 (ver tabla 13).

Tabla 13.

Correlación: "aspectos metodológicos y las competencias básicas"

\begin{tabular}{llc} 
& Coeficiente de correlación & $\begin{array}{c}\text { Competencias } \\
\text { básicas }\end{array}$ \\
Aspectos & $\begin{array}{l}\text { Spearman } \\
\text { metodológicos }\end{array}$ & 0,256 \\
\cline { 2 - 3 } & Sig. (bilateral) & 0,022 \\
\cline { 2 - 3 } & $\mathrm{N}$ & 80 \\
\hline
\end{tabular}

Nota. Elaboración propia.

\section{Hipótesis 4.}

H4a: Existe relación significativa entre los "aspectos académicos del plan curricular" con la "formación por competencias genéricas" de "estudiantes del décimo ciclo de la Escuela Profesional de Administración", UNMSM, 2017-2018.

H4a0: No existe relación significativa entre los "aspectos académicos del plan curricular" con "la formación por competencias genéricas" de los "estudiantes del décimo ciclo de la Escuela Profesional de Administración”, UNMSM, 20172018

\section{Contrastación de Hipótesis 4 (ver tabla 14).}

Tabla 14.

Correlación: "aspectos académicos y las competencias genéricas"

\begin{tabular}{llc} 
& $\begin{array}{c}\text { Competencias } \\
\text { genéricas }\end{array}$ \\
Aspectos & $\begin{array}{l}\text { Soeficiente de correlación } \\
\text { académicos }\end{array}$ & 0,309 \\
\cline { 2 - 3 } & Sig. (bilateral) & 0,005 \\
\cline { 2 - 3 } & $\mathrm{N}$ & 80 \\
\hline
\end{tabular}

Nota. Elaboración propia.

\section{Hipótesis 5.}

H5a: Existe relación significativa entre la "formación laboral del plan curricular" con la "formación por competencias genéricas" de "estudiantes del décimo ciclo de la Escuela
Profesional de Administración", UNMSM, 20172018.

H5a0: No existe relación significativa entre la "formación laboral del plan curricular" con la "formación por competencias genéricas" en los "estudiantes del décimo ciclo de la Escuela Profesional de Administración", UNMSM, 20172018.

\section{Contrastación de Hipótesis 5 (ver tabla 15).}

\section{Tabla 15.}

Correlación: "formación laboral y las competencias genéricas"

\begin{tabular}{llc} 
& $\begin{array}{c}\text { Coeficiente de correlación } \\
\text { Spearman }\end{array}$ & $\begin{array}{c}\text { Competencias } \\
\text { genéricas }\end{array}$ \\
Formación & 0,007 \\
\cline { 2 - 3 } & Sig. (bilateral) & 0,954 \\
\cline { 2 - 3 } & $\mathrm{N}$ & 80 \\
\hline
\end{tabular}

Nota. Elaboración propia.

\section{Hipótesis 6.}

H6a: Existe relación significativa entre los "aspectos metodológicos del plan curricular" con la "formación por competencias genéricas" de "estudiantes del décimo ciclo de la Escuela Profesional de Administración", UNMSM, 20172018.

H6a0: No existe relación significativa entre los "aspectos metodológicos del plan curricular" con la "formación por competencias genéricas" en los "estudiantes del décimo ciclo de la Escuela Profesional de Administración”, UNMSM, 2017-2018.

\section{Contrastación de Hipótesis 6 (ver tabla 16).}

\section{Tabla 16.}

Correlación: "aspectos metodológicos y las competencias genéricas"

\begin{tabular}{llc} 
& $\begin{array}{c}\text { Coeficiente de correlación } \\
\text { Aspectos } \\
\text { metodológicos }\end{array}$ & $\begin{array}{c}\text { Competencias } \\
\text { genéricas }\end{array}$ \\
\cline { 2 - 3 } & Sig. (bilateral) & 0,036 \\
\cline { 2 - 3 } & $\mathrm{N}$ & 0,751 \\
\hline
\end{tabular}

Nota. Elaboración propia.

\section{Hipótesis 7.}

H7a: Existe relación significativa entre los "aspectos académicos del plan curricular" con la "formación por competencias específicas" de "estudiantes del décimo ciclo de la Escuela 
Profesional de Administración”, UNMSM, 20172018.

H7a0: No existe relación significativa entre los "aspectos académicos del plan curricular" con la "formación por competencias específicas" de "estudiantes del décimo ciclo de la Escuela Profesional de Administración", UNMSM, 2017-2018.

\section{Contrastación de Hipótesis 7 (ver tabla 17).}

Tabla 17.

Correlación: "aspectos académicos y las competencias específicas"

\begin{tabular}{llc} 
& $\begin{array}{c}\text { Coeficiente de correlación } \\
\text { Aspectos } \\
\text { académicos }\end{array}$ & $\begin{array}{c}\text { Compecíficas } \\
\text { Spearman }\end{array}$ \\
\cline { 2 - 3 } & Sig. (bilateral) & 0,286 \\
\cline { 2 - 3 } & $\mathrm{N}$ & 0,010 \\
\hline
\end{tabular}

Nota. Elaboración propia.

\section{Hipótesis 8}

H8a: Existe relación significativa entre la "formación laboral del plan curricular" con la "formación por competencias específicas" de "estudiantes del décimo ciclo de la Escuela Profesional de Administración", UNMSM. 2017-2018.

H8a0: No existe relación significativa entre la "formación laboral del plan curricular" con la "formación por competencias específicas" en los "estudiantes del décimo ciclo de la Escuela Profesional de Administración", UNMSM, 2017-2018.

Contrastación de Hipótesis 8 (ver tabla 18).

Tabla 18.

Correlación: "formación laboral y las competencias específicas"

\begin{tabular}{llc} 
& Coeficiente de correlación & $\begin{array}{c}\text { Competencias } \\
\text { específicas }\end{array}$ \\
$\begin{array}{l}\text { Formación } \\
\text { laboral }\end{array}$ & Spearman & 0,262 \\
\cline { 2 - 3 } & Sig. (bilateral) & 0,019 \\
\cline { 2 - 3 } & $\mathrm{N}$ & 80 \\
\hline
\end{tabular}

Nota. Elaboración propia.

\section{Hipótesis 9}

H9a: Existe relación significativa entre los "aspectos metodológicos del plan curricular" con la "formación por competencias específicas" de "estudiantes del décimo ciclo de la Escuela Profesional de Administración", UNMSM, 2017-2018.

H9a0: No existe relación significativa entre los "aspectos metodológicos del plan curricular" con la "formación por competencia" de la Escuela Profesional de Administración, UNMSM, 2017-2018.

Contrastación de Hipótesis 9 (ver tabla 19).

Tabla 19.

Correlación: "aspectos metodológicos y las competencias específicas"

\begin{tabular}{llc} 
& $\begin{array}{c}\text { Coeficiente de correlación } \\
\text { Spearman }\end{array}$ & $\begin{array}{c}\text { Competencias } \\
\text { específicas }\end{array}$ \\
\cline { 2 - 3 } metodológicos & Sig. (bilateral) & 0,115 \\
\cline { 2 - 3 } & $\mathrm{N}$ & 0,308 \\
\hline
\end{tabular}

Nota. Elaboración propia.

\section{Hipótesis general}

HG: El plan curricular se relaciona con la "formación por competencias" en los "estudiantes de décimo ciclo de la Escuela Profesional de Administración", UNMSM, 2017-2018.

HG0: El plan curricular no se relaciona con la "formación por competencias" en los "estudiantes de décimo ciclo de la Escuela Profesional de Administración", UNMSM, 2017-2018.

\section{Contrastación General}

El plan curricular se relaciona con la formación por competencias en los "estudiantes de décimo ciclo de la Escuela Profesional de Administración", UNMSM-2018.

Dado que el nivel de significancia es 0.000 menor que 0.05 , se rechaza la hipótesis nula. Existe una correlación significativa y directa en un nivel regular $(\mathrm{r}=0,419)$ entre el plan curricular y la formación por competencias al 95\% de confianza.

El diagrama de dispersión entre el plan curricular y la formación por competencias explica la presencia de una ligera tendencia lineal, asociación y/o correlación directa (ver tabla 20).

\section{Tabla 20.}

Correlación: "plan curricular y la formación por competencias"

\begin{tabular}{llc} 
& \multicolumn{1}{c}{$\begin{array}{c}\text { Formación por } \\
\text { competencias }\end{array}$} \\
\cline { 2 - 3 } Plan curricular & $\begin{array}{l}\text { Coeficiente de correlación } \\
\text { Spearman }\end{array}$ & 0,419 \\
\cline { 2 - 3 } & Sig. (bilateral) & 0,000 \\
\cline { 2 - 3 } & $\mathrm{N}$ & 80 \\
\hline
\end{tabular}

Nota. Elaboración propia. 


\section{DISCUSIÓN}

Existe una valoración media acerca de la percepción del alumnado de administración con respecto al plan curricular. Es decir, los alumnos $(68 \%)$ piensan que de alguna manera el plan curricular ha influenciado en su formación. Según Alejos y Sánchez (2015) mencionan que "en un estudio anterior nos dice que existe una relación positiva moderada (Rho de 0,482 ) entre el plan curricular y el perfil profesional de la carrera de biología de la Universidad Ricardo Palma con un nivel de significancia de $0.000 "$ (p. 124). La percepción de estudiantes del Décimo Ciclo de dicha carrera. Vemos que la valoración dada al plan curricular en este último caso lo relaciona con el perfil de egreso que se desea, el perfil de un profesional se da cuando obtiene determinadas competencias en su formación, lo cual corrobora nuestra propuesta. Por otra parte, Frau (2010) sugiere que "en el desarrollo de programas curriculares haya consejeros para asegurar la competencia adquirida del profesional" (p.182). Isabell Ann (2014) nos dice que "a una mayor duración de la participación del programa de estudio se tiene una relación más fuerte con competencias y actividades extracurriculares desarrolladas" (p. 77).

Los alumnos de administración mayoritariamente $(91,3 \%)$, rescatan que los "aspectos académicos del plan curricular" cumplen medianamente sus objetivos. Alejos y Sánchez (2015) en su estudio concluyen que "existe una relación positiva débil (Rho de 0.391), entre los aspectos académicos del plan curricular y el perfil profesional de la carrera de biología de la Universidad Ricardo Palma con nivel de significancia de 0.000 " (p. 124). La percepción de estudiantes del sexto al Décimo Ciclo. Es decir que para los futuros biólogos en plan curricular no ha transmitido correctamente lo aspectos académicos, como sí lo es para los futuros egresados de administración de la UNMSM. Por otra parte, Mejía (2015) en su estudio se determinó que:

existe pues relación significativa entre el plan curricular y el desempeño académico conceptual, procedimental, actitudinal en este caso de estudiantes de últimos ciclos de las carreras de contabilidad y finanzas de la Universidad Privada de San Martín de Porres - 2014. (pp. 173-174)

Existe una baja valoración con respecto a la formación laboral, la mayoría de alumnos de administración (56.3\%) piensa, que su formación laboral ha sido pobre. Alejos y Sánchez (2015) en su estudio tiene como resultado que "existe una relación positiva débil (Rho de 0.255), entre la formación laboral y el perfil profesional de la carrera de biología de la Universidad Ricardo Palma con un nivel de significancia de 0.024 " (p. 124), la percepción de estudiantes del sexto al Décimo Ciclo. Una relación pobre, es decir, que se tiene que mejorar la formación laboral para tener profesionales competentes. Podemos mencionar a Mejía (2015) que propone "adaptar el currículo como instrumento de las competencias en mercados de trabajo rápidamente cambiantes dando mayor transparencia y efectividad en las transacciones de empleo y capacitación" (p. 174). También sugiere propiciar un mayor acercamiento universidad - empresa, para mejorar la calidad y pertinencia en la formación. De esta manera se pueden realizar convenios con empresas para que los alumnos realicen sus prácticas profesionales.

Los alumnos de administración en su mayoría $(87,5 \%)$, creen que en su carrera no se ha desarrollado mucho el aspecto metodológico. Alejos y Sánchez (2015) en su estudio tiene como resultado que "existe una relación positiva moderada (Rho de 0.416 ), entre aspectos metodológicos y el perfil profesional de la carrera de biología de la Universidad Ricardo Palma con un nivel de significancia de 0.024" (p. 124), la percepción de estudiantes del sexto al Décimo Ciclo. Dada las características de las carreras, se aprecia que los futuros científicos tienen una rigurosa metodología de investigación tal vez por este factor tienen una correlación moderada con el perfil profesional que a su vez cumple ciertas competencias laborales. También mencionamos que Mejía (2015) propone "realizar un plan de acción permanente para sistematizar, dar continuidad y apoyo institucional al proceso de evaluación de la enseñanza a base de competencias" (p. 175).

Existe una valoración baja acerca de la percepción del alumnado con respecto a la formación por competencias. Es decir, los alumnos 
$(71,3 \%)$ piensan que en alguna forma no se han formado competentemente. Solo un $28,8 \%$ de alumnado piensa que medianamente se ha formado competentemente. Sáenz (2010) en su proyecto de tesis nos muestra ciertas competencias comunes exigidas por el mercado para egresados de distintas carreras las cuales son: "compromiso con la calidad, habilidades interpersonales, capacidad de trabajo en equipo, capacidad creativa, capacidad de aprender y actualizarse" (p. 9).

Los alumnos mayoritariamente (55\%), piensan que su formación por competencias básicas ha sido pobre, seguido de un $45 \%$ que piensa que su formación por competencias básicas tiene una valoración media. Bycroft (2011) nombra las competencias básicas que los investigadores necesitan para practicar la investigación: "Estas competencias básicas se pueden dividir en cuatro áreas: Competencias personales, competencias de conocimiento y habilidad, competencias situacionales y organizacionales, y competencias metodológicas" (p. 231). Granados (2013) menciona que "la investigación demuestra que del total de estudiantes de la escuela de administración de turismo el 54.17\% tiene una buena percepción sobre las competencias básicas" (p. 151). Un resultado distinto a pesar de que ambas carreras, administración y administración de turismo pertenece a la misma facultad de la Universidad Nacional Mayor de San Marcos.

Los alumnos mayoritariamente (57.5\%) piensan que su formación por competencias genéricas ha sido pobre, seguido de un $42.5 \%$ que piensa que su formación por competencias genéricas tiene una valoración media. Granados (2013) menciona que "la investigación demuestra que del total de estudiantes de la escuela de administración de turismo el $67.71 \%$ tiene una buena percepción sobre las competencias genéricas" (p. 151), a diferencia de los alumnos de administración donde el valor de apreciación es más pobre, a pesar de que siendo la misma facultad al parecer tiene otra plana docente.

Los alumnos en general (87.5\%) perciben que han recibido una baja formación por competencias específicas. Solo un $12.5 \%$ afirma que ha recibido una valoración media en formación por competencias específicas. Cross (2010) realiza "una investigación para desarrollar un modelo de competencia válido y práctico con potencial de impacto para mejorar y empoderar el liderazgo y gestión empresarial (Modelo de Rueda de competencia)" (p.173). Las participantes pasarán por esta capacitación y adquirirán estas competencias tan importantes en la administración de empresas. Granados (2013) menciona "la investigación demuestra que del total de estudiantes de la escuela de administración de turismo el $58.33 \%$ tiene una buena percepción sobre las competencias específicas" (p. 151), frente a la percepción de los alumnos de administración que perteneciendo a la misma facultad como sabemos no comparten la misma plana docente, lo cual se debería corregir.

Para realizar la prueba de hipótesis se ha utilizado el coeficiente de Spearman, como estadístico no paramétrico debido a que los datos no siguen una distribución normal.

En la Figura 1, vemos que los datos de las variables (plan curricular y formación por competencias) pueden ajustarse a una recta, con un nivel de correlación significativo.

Los resultados de las hipótesis generales y específicas planteadas nos indican que en algunos casos se acepta la hipótesis nula y en otros casos se rechaza, como vemos en las conclusiones del presente trabajo.

\section{CONCLUSIONES}

Nuestra investigación tiene como conclusión principal mostrar la relación el Plan Curricular logrado en la formación de estudiantes del décimo ciclo que están por egresar de la Escuela de Administración de la UNMSM de los años 2017 y 2018; la tabla 20 de nuestros Resultados y expresada en la tabla 21 , nos muestra:

Existe una correlación significativa y directa en un nivel regular entre la variable bajo las condiciones de Hipótesis, sustentando nuestra posición en los contenidos de Discusión, sostenemos la baja relación (correlación): entre las metodologías empleadas; los aspectos académicos; como la formación laboral de alumnos por egresar (2017-2018). Resumidos en la tabla 22. 


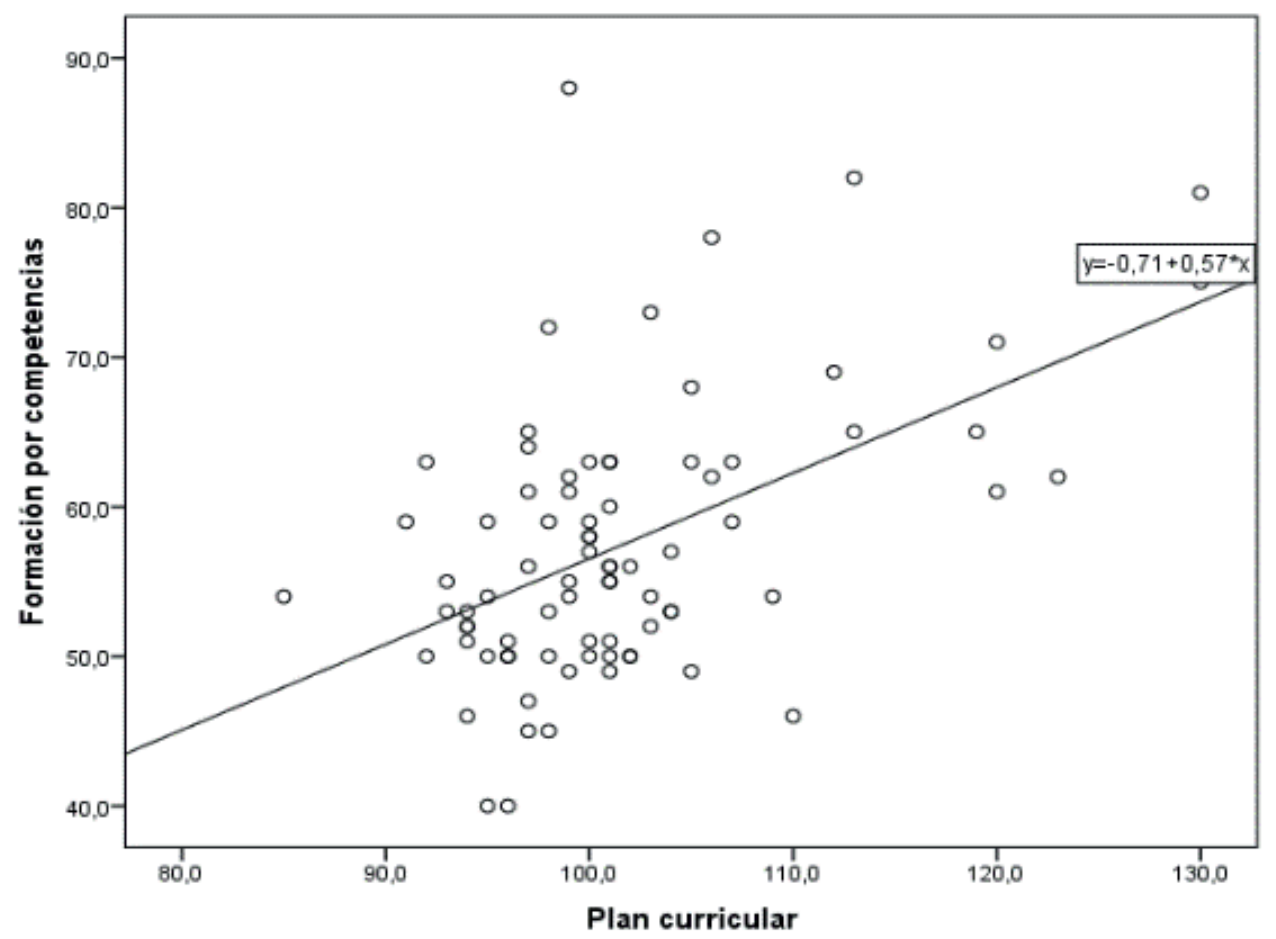

Figura 1. Diagrama de dispersión entre el plan curricular y la formación por competencias.

Elaboración propia

Tabla 21.

Relación de variables (contrastación general)

\begin{tabular}{lcc} 
& $\begin{array}{c}\text { Plan Curricular } \\
\text { Nivel de Significancia }\end{array}$ & Coeficiente de Spearman \\
\hline Formación por Competencias & 0 & 0.419 \\
\hline
\end{tabular}

Nota. Elaboración propia

Tabla 22.

Relación de variables (específicas)

\begin{tabular}{lccc} 
& $\begin{array}{c}\text { Aspectos Académicos } \\
\text { del Plan Curricular }\end{array}$ & $\begin{array}{c}\text { Formación laboral } \\
\text { del Plan Curricular } \\
\text { Coeficiente de Speraman }\end{array}$ & $\begin{array}{c}\text { Metodología del Plan } \\
\text { Curricular }\end{array}$ \\
\hline Formación por competencias básicas & 0.39 & 0.158 & 0.256 \\
Formación por competencias genéricas & 0.309 & 0.007 & 0.036 \\
Formación por competencias especificas & 0.286 & 0.262 & 0.115 \\
\hline
\end{tabular}

Nota. Elaboración propia

\section{BIBLIOGRAFÍA}

Alejos, B. y Sánchez, J. (2015). Tesis de Maestría: "Plan curricular y su relación con el perfil profesional de la carrera de Biología de la Universidad Ricardo Palma”, Lima-Perú, pp.124, 126.

Ayes, G. (2003). "El Diseño Curricular. Informe final de Proyecto de Investigación. ISPETP”, Ciudad de La Habana, Cuba. p. 3. Recuperado de: https://www.monografias.com/trabajos 37/ dimensiones-curriculares/dimensiones-curriculares.shtml

Bycroft, T. (2011). Tesis Doctoral: "Percepciones de expertos en investigación con respecto a las competencias necesarias para practicar una investigación segura con asuntos humanos", Universidad Estatal de Oklahoma, EEUU, p. 231 
Cross, J (2010). “Participación de la paradoja de la competencia: investigación en negocio y educación desarrollando un modelo de competencia de máster de negocio", Capella University, Universidad en Mineápolis, Minnesota, EEUU, p. 173

Frau, R. (2010). Tesis Doctoral: "Los currículos de los programas de maestría de educación en consejería y su relación con las competencias profesionales y multiculturales", Universidad de Puerto Rico, EEUU, p. 182.

Granados, M. (2013), Tesis Doctoral, “Desarrollo competencias laborales y formación de los profesionales en turismo, en la Universidad Nacional Mayor de San Marcos" - ño 2013, Lima - Perú, p. 151

Isabell Ann M. (2014), Tesis Doctoral: "Análisis de las relaciones entre programas / actividades extracurriculares y competencias", Universidad de Claremont, EEUU, p. 77
Larraín, A. y González, L. (2007). “Formación universitaria por competencias", Chile, p.10

Lovatón, P. (2011). Tesis de Maestría: “El Diagnóstico Situacional: herramienta indispensable en la elaboración del currículo para la formación académico-profesional del comunicador social al 2011", Lima-Perú, p. 97

Mejía P. (2015). Tesis Doctoral: "El Plan curricular y el Desempeño académico por competencias de los estudiantes de los últimos ciclos de la escuela profesional de contabilidad y finanzas USMP - año 2014", Lima - Perú. pp.173-175

Sáenz, B. (2010). Tesis de Maestría, "Modelo educativo para la formación de competencias genéricas en estudiantes de la carrera profesional técnica de administración bancaria y su interrelación con las competencias genéricas laborales exigidas por el mercado laboral bancario de Lima Metropolitana en el año 2009 a 2010", Lima - Perú, p. 9 